\title{
Embracing National Cancer Registries for Improved Care of Rare Tumors
}

\author{
David F. Schneider, MD, MS \\ Department of Surgery, K4/738 Clinical Science Center, University of Wisconsin, Madison, WI
}

Adrenocortical carcinoma (ACC) is one of the scarcest but deadliest cancers. According to recent Surveillance, Epidemiology, and End Results data, the incidence in the United States is 0.72 persons per 1 million population's The scarcity of ACC makes it nearly impossible for any single center to amass enough data for any meaningful conclusions. National cancer registries provide large volumes of data related to patient, tumor, and treatment characteristics. In this issue of Annals of Surgical Oncology, Gratian et al. used the National Cancer Data Base (NCDB) to analyze treatment patterns and outcomes for ACC as they relate to hospital volume. ${ }^{1}$ Patients at highvolume centers were more likely to undergo radical resection, lymph node evaluation, and chemotherapy. Despite more aggressive treatment, survival of patients treated at high-volume centers did not differ from that of patients treated at low-volume centers. ${ }^{1}$ This is a well-done study and could never have been done without large national cancer registries such as the NCDB.

Although the NCDB made this study possible, there are some issues with large national cancer databases that this study also brings to light. Gratian and colleagues defined high volume as at least four cases of primary adrenal malignancy annually. ${ }^{1}$ This selective definition might be unnecessary or even misleading for a few reasons. It likely represents the select subset of centers that solely focus on cancer treatment. This definition likely classified many centers performing an overall high volume of adrenal surgery as "low volume" due to the malignancy requirement. Since guidelines rarely recommend biopsy of adrenal

(C) Society of Surgical Oncology 2014

First Received: 18 June 2014;

Published Online: 26 July 2014

D. F. Schneider, MD, MS

e-mail: schneiderd@surgery.wisc.edu masses, the diagnosis of ACC becomes confirmed only at operation. ${ }^{2}$ This methodology likely selected out patients with a preoperative diagnosis of ACC who sought care at superspecialized cancer centers.

Additionally, the NCDB lacks complete data on recurrence. A recent study highlighted the lack of recurrence data in national cancer databases, and the NCDB is not alone. ${ }^{3}$ Even though survival did not differ between highand low-volume centers, might it have affected recurrence rates? Missing or incomplete data also become a problem in any large registry. In the study by Gratian et al., tumor grade was missing in $77 \%$, and clinical stage was missing in $84 \%{ }^{1}$ Although this could lead to bias when comparing-high volume with low-volume centers, imputation techniques can adjust for missing data.

Many would cite these issues and dismiss this work altogether. It is very easy to identify clinical nuances or problematic features with population-level data. Any dataset has its own weaknesses, and I would challenge them to analyze better data for ACC. National cancer registries are not perfect. Perhaps if we could study disease-free survival in ACC, we might find that more aggressive treatment carried out at high-volume centers does affect recurrence.

So what is the take-home message from the study by Gratian et al.? The most striking finding is that despite the most aggressive treatment, done at the highest-volume, specialized centers, ACC remains such a fatal cancer that aggressive treatment and experienced clinicians from highly specialized centers cannot really move the bar in terms of survival. Historically, only $30 \%$ of ACCs were confined to the adrenal gland at diagnosis. ${ }^{4}$ Conventional wisdom suggests that widespread use of high-quality crosssectional imaging would lead to earlier diagnosis of smaller tumors, thereby leading to earlier treatment and improved outcomes. This study and an earlier analysis of the NCDB indicate that this simply is not true. ${ }^{5}$ At the very least, this 
should make us question current practices and begin to think about alternatives. Instead of criticizing these types of studies that use population-level data, I think we should instead focus on improving them. As electronic, automated systems to extract data from medical records improve, so too will the quality of our national cancer databases. The rapid adoption of electronic medical record systems, natural language processing, and increased interoperability of electronic medical record systems will hopefully allow us to capture more complete information and additional variables such as recurrence. ${ }^{6}$

Finally, testing new treatments for rare diseases such as ACC remains challenging, and prospective clinical trials are not always feasible. The consolidation of data on outcomes and treatment is the first step. Newer comparative effectiveness research techniques such as pragmatic clinical trials, simulation, and propensity scores can identify optimal treatment strategies for rare diseases such as ACC. ${ }^{7}$ The study by Gratian et al. indicated dismal outcomes for ACCs despite our most aggressive methods used at the highest-volume centers. Despite the unfavorable biology of ACC, the continued use of big data and its associated comparative effectiveness methodologies along with surgical and medical innovation hopefully can identify different treatments that improve upon our current treatment of ACC.

\section{REFERENCES}

1. Gratian L, Pura J, Dinan M, Reed S, Roman S, Sosa JA. Treatment patterns and outcomes for patients with adrenocortical carcinoma associated with hospital case volume in the United States. Ann Surg Oncol. In press.

2. Chen H, Sippel RS, O’Dorisio MS, Vinik AI, Lloyd RV, Pacak K. The North American Neuroendocrine Tumor Society consensus guideline for the diagnosis and management of neuroendocrine tumors: pheochromocytoma, paraganglioma, and medullary thyroid cancer. Pancreas. 2010;39:775-83.

3. In $\mathrm{H}$, Bilimoria $\mathrm{KY}$, Stewart $\mathrm{AK}$, et al. Cancer recurrence: an important but missing variable in national cancer registries. Ann Surg Oncol. 2014;21:1520-9.

4. Kendrick ML, Lloyd R, Erickson L, et al. Adrenocortical carcinoma: surgical progress or status quo? Arch Surg. 2001;136: 543-9.

5. Bilimoria KY, Shen WT, Elaraj D, et al. Adrenocortical carcinoma in the United States: treatment utilization and prognostic factors. Cancer. 2008;113:3130-6.

6. Hersh WR, Weiner MG, Embi PJ, et al. Caveats for the use of operational electronic health record data in comparative effectiveness research. Med Care. 2013;51(8 Suppl. 3):S30-7.

7. Neuman HB, Greenberg CC. Comparative effectiveness research: opportunities in surgical oncology. Semin Radiat Oncol. 2014;24: 43-8. 